OPEN ACCESS

Edited by:

Ake Sjoholm,

University of South Alabama,

United States

Reviewed by:

Jan Brož,

Charles University, Czechia

Joseph Aloi,

Wake Forest Baptist

Medical Center, United States

${ }^{*}$ Correspondence:

Elif I. Ekinci

elif.ekinci@unimelb.edu.au

tThese authors have contributed equally to this work.

Specialty section: This article was submitted to Diabetes,

a section of the journal Frontiers in Endocrinology

Received: 12 December 2016 Accepted: 02 May 2017

Published: 13 June 2017

Citation:

Tran TIT, Pease A, Wood AJ, Zajac JD, Mårtensson J, Bellomo R

and Ekinci El (2017) Review of

Evidence for Adult Diabetic Ketoacidosis Management Protocols.

Front. Endocrinol. 8:106. doi: 10.3389/fendo.2017.00106

\section{Review of Evidence for Adult Diabetic Ketoacidosis Management Protocols}

Tara T. T. Tran ${ }^{1 \dagger}$, Anthony Pease ${ }^{1 \dagger}$, Anna J. Wood ${ }^{1}$, Jeffrey D. Zajac ${ }^{1,2}$, Johan Mårtensson ${ }^{3}$, Rinaldo Bellomo ${ }^{3}$ and Elif I. Ekinci',2,4*

${ }^{1}$ Department of Endocrinology, Austin Health, Melbourne, VIC, Australia, ${ }^{2}$ Department of Medicine, Austin Health, University of Melbourne, Melbourne, VIC, Australia, ${ }^{3}$ Department of Intensive Care, Austin Health, Melbourne, VIC, Australia, ${ }^{4}$ Menzies School of Health Research, Darwin, NT, Australia

Background: Diabetic ketoacidosis (DKA) is an endocrine emergency with associated risk of morbidity and mortality. Despite this, DKA management lacks strong evidence due to the absence of large randomised controlled trials (RCTs).

Objective: To review existing studies investigating inpatient DKA management in adults, focusing on intravenous (IV) fluids; insulin administration; potassium, bicarbonate, and phosphate replacement; and DKA management protocols and impact of DKA resolution rates on outcomes.

Methods: Ovid Medline searches were conducted with limits "all adult" and published between "1973 to current" applied. National consensus statements were also reviewed. Eligibility was determined by two reviewers' assessment of title, abstract, and availability.

Results: A total of 85 eligible articles published between 1973 and 2016 were reviewed. The salient findings were (i) Crystalloids are favoured over colloids though evidence is lacking. The preferred crystalloid and hydration rates remain contentious. (ii) IV infusion of regular human insulin is preferred over the subcutaneous route or rapid acting insulin analogues. Administering an initial IV insulin bolus before low-dose insulin infusions obviates the need for supplemental insulin. Consensus-statements recommend fixed weight-based over "sliding scale" insulin infusions although evidence is weak. (iii) Potassium replacement is imperative although no trials compare replacement rates. (iv) Bicarbonate replacement offers no benefit in DKA with $\mathrm{pH}>6.9$. In severe metabolic acidosis with $\mathrm{pH}<6.9$, there is lack of both data and consensus regarding bicarbonate administration. (v) There is no evidence that phosphate replacement offers outcome benefits. Guidelines consider replacement appropriate in patients with cardiac dysfunction, anaemia, respiratory depression, or phosphate levels $<0.32 \mathrm{mmol} / \mathrm{L}$. (vi) Upon resolution of DKA, subcutaneous insulin is recommended with IV insulin infusions ceased with an overlap of 1-2 $\mathrm{h}$. (vii) DKA resolution rates are often used as end points in studies, despite a lack of evidence that rapid resolution improves outcome. (viii) Implementation of DKA protocols lacks strong evidence for adherence but may lead to improved clinical outcomes.

Conclusion: There are major deficiencies in evidence for optimal management of DKA. Current practice is guided by weak evidence and consensus opinion. All aspects of DKA management require RCTs to affirm or redirect management and formulate consensus evidence-based practice to improve patient outcomes.

Keywords: diabetic ketoacidosis, diabetes, insulin, rehydration, hypoglycemia, hypokalemia, metabolic acidosis, protocol 


\section{INTRODUCTION}

\section{Background}

Diabetic ketoacidosis (DKA) is characterised by the triad of hyperglycemia, ketosis, and metabolic acidosis. This results from a relative or absolute deficiency of insulin and an excess of counter-regulatory hormones including glucagon, cortisol, catecholamines, and growth hormones leading to hyperglycemia, glycosuria, dehydration, and hyperosmolarity of varying severity (Table 1) (1,2). Glycosuria induces an osmotic diuresis, which results in significant deficits in fluid and electrolytes including sodium, potassium, calcium, magnesium, chloride, and phosphate (3). Dehydration and hyperglycemia results in hypertonicity and an efflux of water from the intracellular space to the hypertonic extracellular space. There is also a potassium efflux from the intracellular space, aggravated by acidosis, lack of effective insulin action, and breakdown of intracellular proteins (3) (Figure 1).

Diabetic ketoacidosis more often complicates type 1 rather than type 2 diabetes mellitus and carries the risk of significant morbidity and mortality $(1,4,5)$. Despite evolving practice, there are increasing numbers of hospital admissions for DKA and hyperosmolar hyperglycaemic state (HHS) $(1,6,7)$. DKA is associated with mortality rates as high as $5-9 \%$ in the elderly and in patients with severe comorbidities $(1,8)$. Though mortality from DKA is more often attributable to severe underlying illness and comorbidities (1), DKA itself is a hypercoagulable state resulting in potentially fatal complications including stroke, myocardial infarction, and disseminated intravascular coagulation $(9,10)$. Management involves rehydration, correction of electrolyte derangements; particularly hypokalaemia, administration of insulin, correction of metabolic acidosis, and treatment of precipitants such as infection, pancreatitis, trauma, and myocardial infarction (11-13). Complications of DKA management include pulmonary venous congestion and severe electrolyte imbalance. Cerebral oedema represents a major potential complication, although this has largely been demonstrated in children.

\section{Aims of Review}

This review is intended to assist those writing and utilising DKA management protocols in adults to appreciate deficits in current knowledge and to draw attention to areas that may benefit from future research.

We reviewed the original studies considering key elements of inpatient management of DKA including the choice of intravenous

TABLE 1 | Definitions of severity of diabetic ketoacidosis (DKA).

\begin{tabular}{|c|c|c|c|}
\hline & Mild DKA & Moderate DKA & Severe DKA \\
\hline Arterial pH & $7.25-7.3$ & 7.0 to $<7.24$ & $<7.0$ \\
\hline $\begin{array}{l}\text { Serum bicarbonate } \\
(\mathrm{mmol} / \mathrm{L})\end{array}$ & 15-18 & 10 to $<15$ & $<10$ \\
\hline Anion gap & $>10$ & $>12$ & $>10$ \\
\hline Mental status & Alert & Alert/drowsy & Stupor/coma \\
\hline
\end{tabular}

Adapted from American Diabetes Association 2009 consensus statement. In all cases, plasma glucose is >14 mmol/L (>250 mg/dL), urine, and serum ketones are positive and effective osmolality is variable. fluids and rates of replacement; insulin infusion rates, and routes of administration; potassium replacement rates; and the role of bicarbonate and phosphate replacement.

As many institutions use protocol driven management, we considered the role of protocols by evaluating available audits of such documents to determine whether these protocols significantly alter clinician decisions, patient outcomes, and hospitalisation duration. We also addressed the correlation of DKA correction rate on patient outcomes.

\section{METHODS}

From January 3, 2015, until April 11, 2016, two review authors: AP and TT performed Ovid Medline searches combining Subject Headings (Diabetic Ketoacidosis.mp. or Diabetic Ketoacidosis/), with key words of different DKA management aspects, with the applied limits: "all adult" (19 plus years), published from "1973 to current” (Figure 2).

\section{RESULTS}

Ovid Medline searches yielded 1,432 articles in addition to those accessible from reference lists of national consensus statements. Eligibility was determined by two reviewers' assessment of title, abstract, and full-text/print availability through affiliations with Austin Health's and the University of Melbourne's libraries. 85 articles published between 1973 and 2016 were considered eligible and were reviewed (Figure 2).

\section{Hydration}

Patients with DKA experience osmotic diuresis, resulting in hyperosmolar intracellular dehydration (14). Fluid deficits may be up to $10 \%$ of total body weight $(1,5)$. There is also an accumulation of $\beta$-hydroxybutyrate and acetoacetate, which results in a high anion gap metabolic acidosis (1). Prompt rehydration is vital to restore circulating volume and tissue perfusion, clear ketones, and correct electrolyte imbalances $(1,5,14)$. Independent of insulin therapy, hydration alone restores circulatory volume and tissue perfusion; improves glycaemic control and acid base balance, and reduces counterregulatory hormones $(5,14)$.

\section{Hydration - Choice of Fluids}

Isotonic fluids have been established as the preferred fluid choice since a pivotal study in 1958 compared with hypotonic, isotonic, and hypertonic fluids in patients with severe DKA. These authors found that hypertonic fluids may be detrimental due to worsening of hyperosmolarity, hypernatraemia, and hyperchloraemia, while hypotonic fluids were noted to cause diuresis (3).

In 2013, a Cochrane review of 78 randomised controlled trials (RCTs) assessed the effect of colloids compared to crystalloids for fluid resuscitation in critically unwell patients with trauma, burns or in the post-operative period (15). Crystalloids were preferred as colloids were considerably more expensive and offered no survival benefit over crystalloids (15). However, specifically hydroxyethyl starch crystalloids were associated with a significant increase in mortality and acute kidney injury, and it was concluded that they should be avoided (14-19). It is important to 


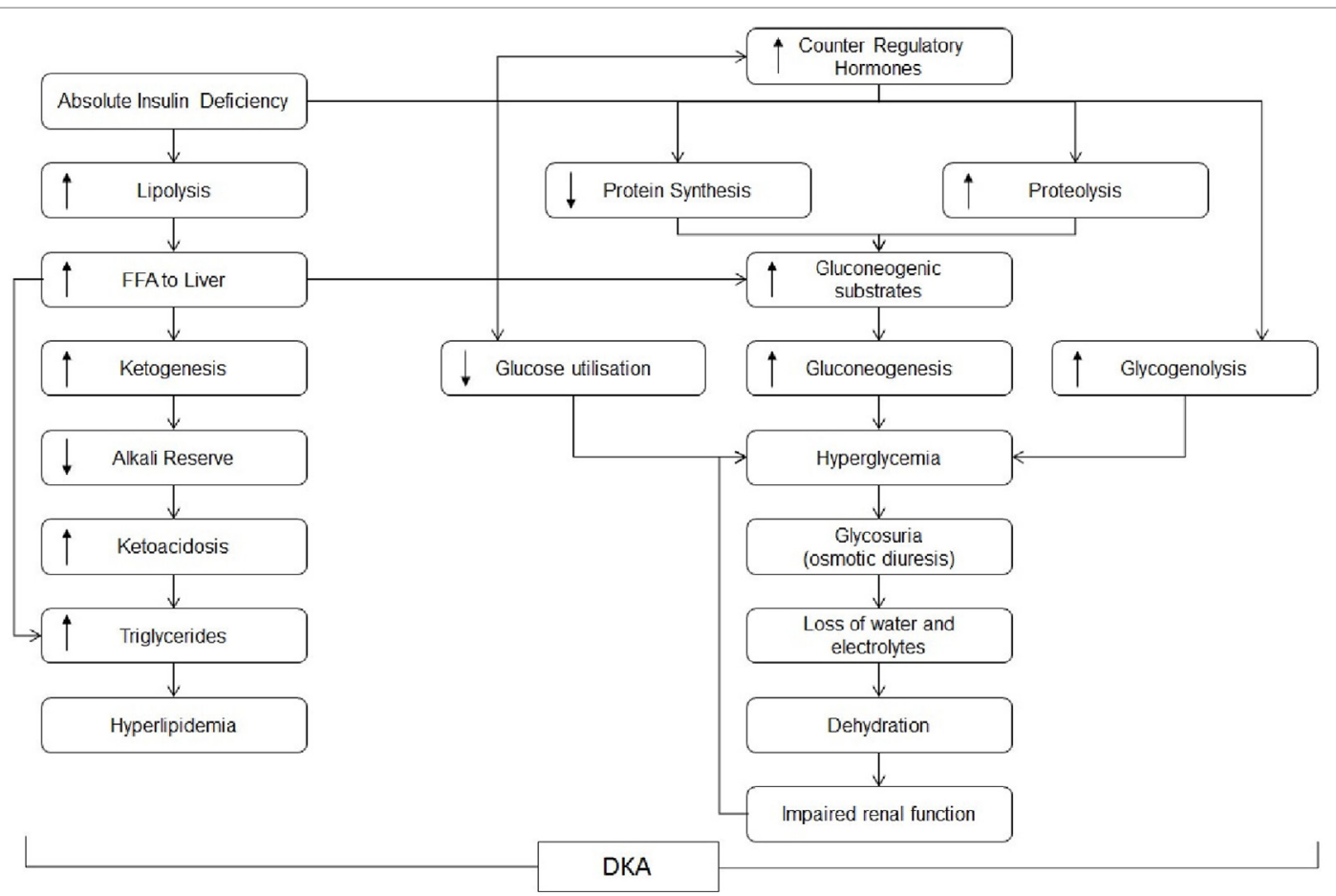

FIGURE 1 | Pathogenesis of diabetic ketoacidosis.

note that none of the included studies specifically considered these fluids in the setting of DKA.

The optimal crystalloid solution to use in DKA is unclear (Table 2). Among crystalloids, normal saline (0.9\% sodium chloride) has come under scrutiny due to concerns raised over hyperchloraemic metabolic acidosis, as well as a possible propensity for oliguria, prolonged acidosis, and coagulopathy $(1,5,14$, 16-18, 20-25).

During the recovery phase of DKA, hyperchloraemia tends to develop because of preferential excretion of ketones during rehydration and improved renal perfusion, resulting in a raised anion gap metabolic acidosis (14). It is proposed that rehydration with normal saline may contribute to hyperchloraemia and a hyperchloraemic metabolic acidosis with a persisting base deficit and may cause renal vasoconstriction and decreased glomerular filtration rate $(14,27)$. Alternatively, this acidosis may represent a physiological response to resolving DKA rather than a result of the hydration fluid itself $(17,20-23,28)$.

In the DKA-specific setting, a small, randomised, doubleblinded, prospective trial $(n=45)$ compared the effects of fluid resuscitation in patients with DKA using Plasma-Lyte (a balanced electrolyte solution) and normal saline and concluded that balanced electrolyte solutions resulted in lower serum chloride and higher bicarbonate levels consistent with prevention of hyperchloraemic metabolic acidosis (16). However, there was no difference in clinical outcomes (16) (Table 2).

A similar conclusion was drawn in a small three centre retrospective study $(n=23)$ which compared Plasma-Lyte to normal saline as fluid resuscitation in DKA (14). This study was limited by its patient numbers with difficulty matching cases to controls.
The only other RCT was also small, studied different crystalloids in DKA, comparing patients who received Ringer's lactate (a balanced electrolyte solution) $(n=28)$ with patients who received normal saline $(n=29)$. In this study, Ringer's lactate offered no significant superiority in time to normalisation of $\mathrm{pH}$ and took a significantly longer time to achieve a blood glucose concentration $\leq 14 \mathrm{mmol} / \mathrm{L}$ (26). A proposed mechanism for this delay in control of blood glucose level was that lactate from Ringer's solution provided excess substrate for ongoing gluconeogenesis (26). Using the 2006 (and then the 2009 post hoc) American Diabetes Association (ADA) definitions for resolution of DKA, fluid selection made no statistically significant difference in time to resolution (26).

Another concern regarding normal saline is saline-induced oliguria. In the DKA context, a RCT comparing Plasma-Lyte to normal saline found cumulative urine output to be lower at 4-6 h in the normal saline group (14).

Balanced electrolyte solutions have insufficient potassium content when used alone and are not available with adequate premixed potassium (7). For this reason, the United Kingdom National Institute for Health Care Excellence (NICE) and the ADA guidelines favour continued use of normal saline (29).

From the available evidence, we conclude that there is no evidence for significant superiority between crystalloid and colloid solutions, while noting that hydroxyethyl starch solutions are best avoided due to risk of mortality and acute kidney injury.

There is currently insufficient evidence for superiority amongst crystalloids, with very weak evidence to suggest that balanced electrolyte solutions may reduce the risk of hyperchloremic metabolic acidosis, though this has not translated to any clinical 


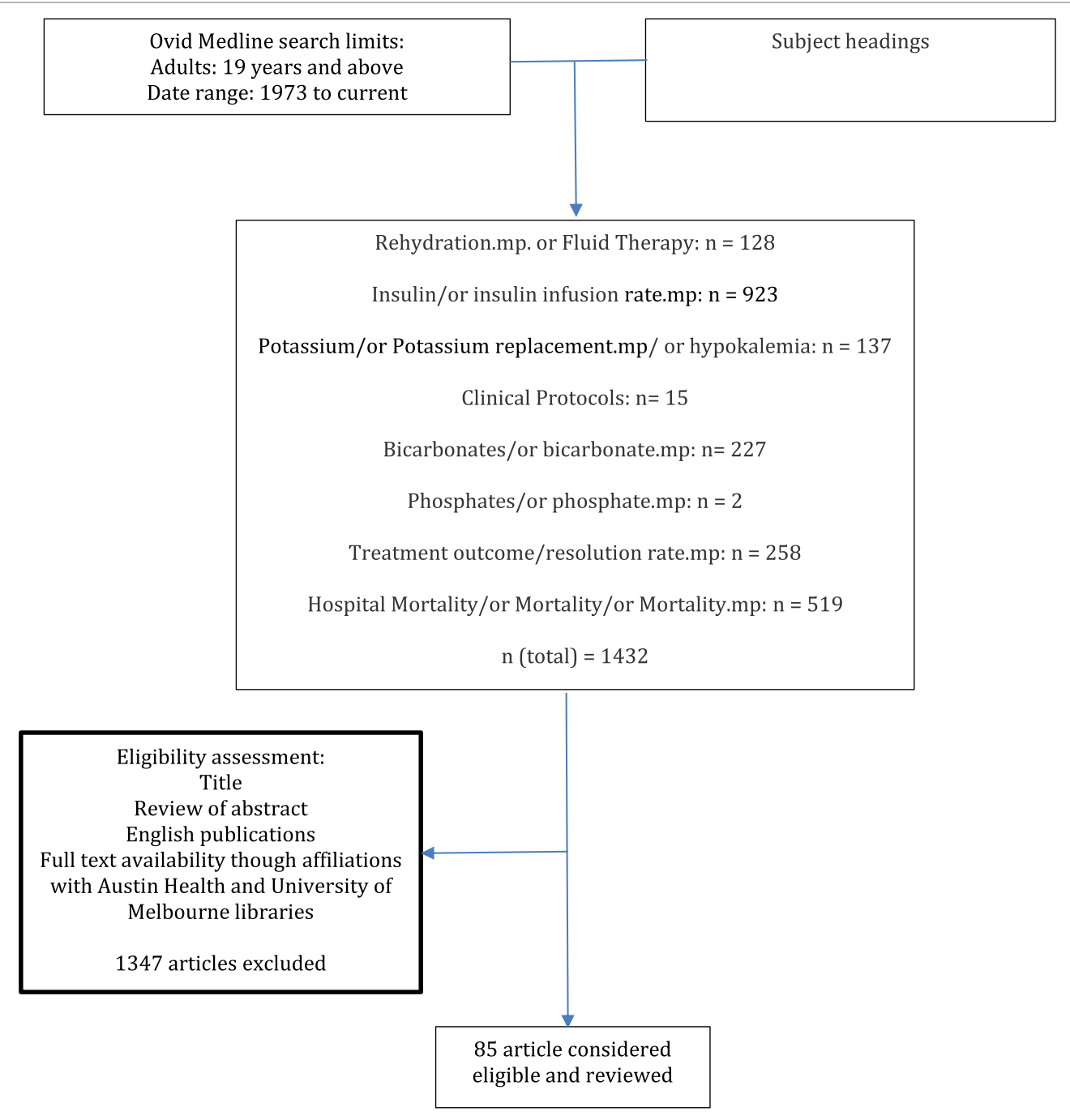

FIGURE 2 | Study selection flow diagram.

significance in patient outcomes in DKA. Normal saline is cheap and familiar to all clinicians and therefore can be used as the fluid of choice (Figure 3).

\section{Hydration-Rate of Replacement}

The rationale for intensive hydration in DKA is to avoid hypoperfusion, correct marked hyperglycaemia and hyperosmolarity, and improve responses to insulin therapy (1). Alternatively, the rationale for slower rehydration is to reduce the risks associated with correcting the hyperosmolar state too rapidly $(30,31)$ thus decreasing the potential risk of cerebral oedema $(31,32)$ as reported in children $(32,33)$.

Most studies of hydration rate take place in the intensive care setting making use of central venous pressure monitoring as opposed to general ward-based management. The latter is more common in the Australian context (22, 28, 34, 35).

Conservative hydration in relatively small numbers of open label studies revealed no adverse events, although the selection of patients was tightly regulated and may not be generalisable
$(22,23,28,35)$. In 1989 , a prospective analysis $(n=28)$ assessed aggressive resuscitation at $1,000 \mathrm{~mL} / \mathrm{h}$ for $4 \mathrm{~h}$ followed by $500 \mathrm{~mL} / \mathrm{h}$ for $4 \mathrm{~h}$, compared to rehydration at half this rate (22). The study concluded that a slower rate of rehydration still led to prompt recovery, lack of any harmful effects, and had a significant reduction in the overall cost of medical therapy (22). This principle was reiterated by another, also prospective randomised controlled study with limited numbers $(n=27)$, comparing the effect of hydration using a rate of $1,000 \mathrm{~mL} / \mathrm{h}$ versus a rate of $500 \mathrm{~mL} / \mathrm{h}$ of normal saline, which concluded there was no significant difference in rate of resolution of biochemical derangements (36).

The major concern with rapid hydration is cerebral oedema which is seen in the paediatric population (33) and rarely reported in adult literature. There have been limited numbers of prospective trials, although given the rarity of this outcome, these have been inadequately powered $(16,20-23,28,35)$. Others have argued that rapid hydration is not a concern as cerebral oedema, as demonstrated by CT imaging of the brain, may be present prior 
TABLE 2 | Choice of crystalloids for rehydration in adult patients with diabetic ketoacidosis.

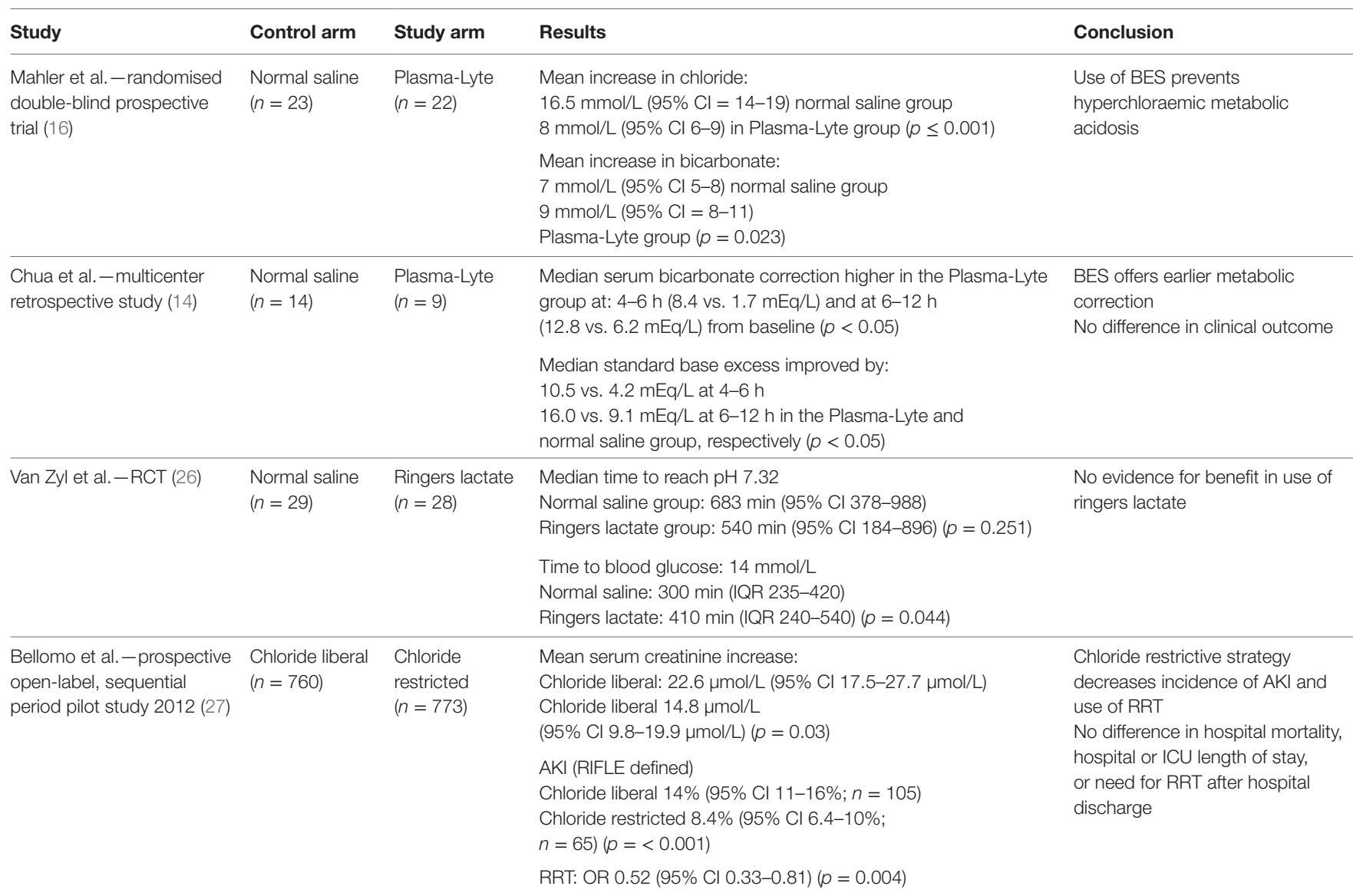

Chloride liberal: normal saline, $4 \%$ succinylated gelatine solution, and $4 \%$ albumin.

Chloride restricted: Hartmann's solution, balanced electrolyte solution (Plasma-Lyte 148), 20\% albumin.

$R R T$, renal replacement therapy; $R C T$, randomised controlled trial.

to hydration and relates to the severity of initial acidosis rather than medical intervention $(31,34)$.

Pulmonary venous congestion is another potential complication of DKA management, although there is minimal evidence for this occurrence. Assessing extravascular lung water and colloid-osmotic pressure in a case series from 1988 did not reveal any tendency towards pulmonary venous congestion, despite large total infused volumes of $6 \pm 1.01 \mathrm{~L}$ (35).

The ADA consensus statement in 2009 recommended that in the absence of cardiac compromise, normal saline is to be infused at a rate of $15-20 \mathrm{~mL} / \mathrm{kg}$ body weight or at $1-1.5 \mathrm{~L}$ during the first hour (1). Determination of subsequent rates should be dependent on patient haemodynamic and hydration status and serum electrolyte levels (1). Neither studies nor guidelines addressed the issue of fluid replacement in obese patients with DKA.

In patients with normal or high corrected serum sodium levels, the recommended rehydration fluid is $1 / 2$ normal saline $(0.45 \%$ sodium chloride) given at $250-500 \mathrm{~mL} / \mathrm{h}$, whilst the recommended choice of rehydration fluid for patients with low corrected serum sodium is still normal saline, infused at the same rate (1). In either instance, fluid deficits should be corrected within the initial 24-h period (1). NICE guidelines recommend hydration at rates which are "not too rapid" except in cases of circulatory collapse (29).

The currently available evidence is very weak and suggests slow hydration rates result in equal management outcomes, with no consensus regarding optimal rates.

\section{Potassium Replacement-Dose and Rates}

Patients with DKA are often found to initially have mild to moderate hyperkalaemia, despite a total body deficit of potassium (1). The initiation of insulin causes an intracellular shift of potassium and lowers the potassium concentration potentially resulting in severe hypokalaemia. Hence patients with serum potassium levels $<3.3 \mathrm{mmol} / \mathrm{L}$ need initial management with fluid resuscitation and potassium replacement, whilst delaying commencement of insulin until after potassium levels are above $3.3 \mathrm{mmol} / \mathrm{L}$, to avoid cardiac arrhythmias, arrest, and respiratory muscle weakness (2).

Published trials use a range of potassium replacement rates from 10 to $40 \mathrm{mmol} / \mathrm{h}$, which have not been reported to lead to adverse events. However, it is noted that potassium infusion rates and potential complications from over-replacement of potassium were not always analysed as outcomes (1, 5, 24, 37-40). These studies were not designed to determine optimal rates of potassium 
DIABETIC KETOACIDOSIS FLOW-CHART (ADULTS / AUSTIN HEALTH) ** Contact Endocrinology about all patients with DKA **

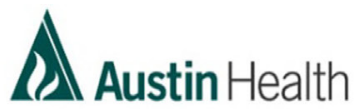

Evaluation: Search for precipitant is key

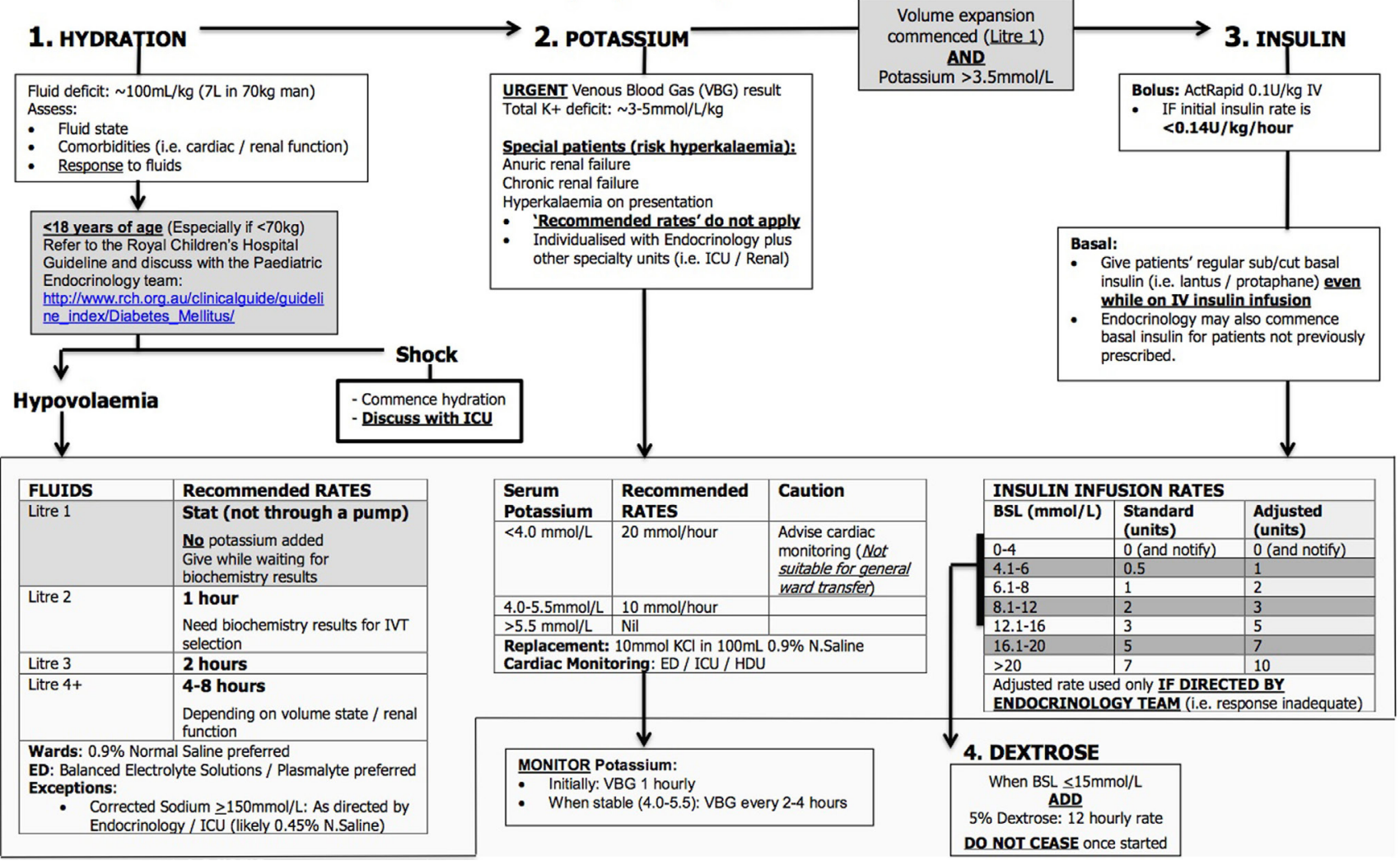

FIGURE 3 | Protocol for management of diabetic ketoacidosis at our centre.

replacement or the required care settings and cardiac monitoring associated with different rates of potassium replacement during treatment of DKA, although it has been recommended that hypokalaemia (below $3.5 \mathrm{mmol} / \mathrm{L}$ ) warrants admission to a high dependency unit (HDU) or equivalent (5).

Of note, the ADA guidelines suggest the addition of 20-30 mmol of potassium in each litre of infusion fluid to maintain target "normokalaemia" defined as a potassium of $4-5 \mathrm{mmol} / \mathrm{L}$ $(1,5)$. This is in contrast to the Joint British Diabetes Societies' guideline recommending a replacement dose of potassium of $40 \mathrm{mmol}$, premixed in infusion fluid, if serum potassium is less than $5.5 \mathrm{mmol} / \mathrm{L}$ and the patient is passing urine, whilst faster replacement is to be guided by local protocols (5).

Currently, there is lack of evidence guiding the ideal potassium replacement rate, the associated necessary cardiac monitoring and care settings with guidelines recommending rates between 20 and $40 \mathrm{mmol}$ in $1 \mathrm{~L}$ of rehydration fluid.

\section{Bicarbonate}

Significant controversy surrounds the role of bicarbonate replacement in DKA. Some argue that correcting ketosis with insulin is adequate for reversing acidosis $(1,2,7,41)$. Others argue that bicarbonate therapy is warranted given the complications of severe metabolic acidosis $(1,2)$ (Table 3$)$.
Small randomised controlled studies demonstrate that bicarbonate can improve acidosis resolution transiently in DKA with pH 6.9-7.1 $(14,45)$ although this did not translate to improvement in morbidity, mortality, or time to recovery from hyperglycaemia, ketoacidosis, cardiac, or neurological outcomes $(1,2,45)$. Conversely, bicarbonate replacement has its own associated complications $(1,14)$ (Table 3). Furthermore, there have been no prospective randomised studies investigating the impact of administering bicarbonate in patients with $\mathrm{pH}<6.9$ (2).

Due to the known complications of severe acidosis (Table 3 ), the ADA consensus guidelines that recommend patients with $\mathrm{pH}<6.9$ should be administered $100 \mathrm{mmol}$ of sodium bicarbonate with $20 \mathrm{mmol}$ potassium chloride/h for $2 \mathrm{~h}$ until venous $\mathrm{pH}$ is $>7.0(1,2)$.

It appears clear that bicarbonate replacement offers no benefit when $\mathrm{pH}>6.9$. For cases of $\mathrm{pH}<6.9$, whilst there is lack of evidence, there is recommendation based only on expert opinion that replacement should be considered.

\section{Phosphate}

In DKA, serum phosphate often appears normal or even increased, although there is a total body deficit of phosphate, typically up to $1.0 \mathrm{mmol} / \mathrm{kg}$ body weight which further decreases with insulin 
TABLE 3 | Complications associated with biochemical derangement and their correction.

\begin{tabular}{|c|c|}
\hline Severe hypokalemia & Potassium over-replacement \\
\hline $\begin{array}{l}\text { Severe muscle weakness or } \\
\text { rhabdomyolysis }\end{array}$ & Muscle weakness and paralysis \\
\hline Cardiac conduction abnormalities & Cardiac conduction abnormalities \\
\hline Fatal arrhythmias (VT, VF) & $\begin{array}{l}\text { Fatal arrhythmias (VT, VF, sinus arrest, } \\
\text { asystole) }\end{array}$ \\
\hline Severe metabolic acidosis & Bicarbonate replacement \\
\hline Impaired myocardial contractility & Hypokalaemia \\
\hline Cerebral vasodilation & Decreased tissue oxygen uptake (1) \\
\hline Gastrointestinal complications & Cerebral oedema in children $(1,14)$ \\
\hline Coma $(1,2)$ & $\begin{array}{l}\text { Paradoxical worsening of ketosis in } \\
\text { adults (14) }\end{array}$ \\
\hline Severe phosphate deficiency & Phosphate over-replacement \\
\hline $\begin{array}{l}\text { Respiratory and skeletal muscle } \\
\text { weakness, Haemolytic anaemia } \\
\text { and poor cardiac contractility }(2,41)\end{array}$ & Hypocalcaemia (1) \\
\hline
\end{tabular}

VT, ventricular tachycardia; VF, ventricular fibrillation.

therapy $(1,2)$. Complications of phosphate deficiency are uncommon but may occur in severe cases $(2,41)$ (Table 3 ).

Prospective randomised studies demonstrated that phosphate replacement offers no improvement to DKA outcomes (1). Acknowledging this, the ADA consensus statement suggests that phosphate replacement with $20-30 \mathrm{mmol}$ of potassium phosphate added to replacement fluid may be appropriate in patients with cardiac dysfunction, anaemia, respiratory depression, or patients with phosphate levels $<3.2 \mathrm{mmol} / \mathrm{L}$ (1). This contrasts against NICE guidelines that recommend against phosphate replacement (29).

\section{Insulin Administration}

Once hypokalaemia is corrected and hydration commenced, insulin should be administered to halt lipolysis, ketogenesis, and correct hyperglycaemia. Regular insulin is favoured over insulin analogues. The current mainstay of insulin therapy in DKA is continuous intravenous infusion for its rapid onset and ease of dose titration $(1,2)$. Some institutions require intravenous insulin infusions to be managed in the intensive care setting and thus some advocate for the use of subcutaneous or intramuscular injections in order to avoid an intensive care admission (1, 46-48).

\section{Insulin-Choice of Insulin}

In 1987, Storms et al established that regular human insulin was superior to porcine insulin due to faster resolution of DKA (49), and for decades regular human insulin has remained the insulin of choice in DKA management. This convention has been challenged by the emergence of short and ultrashort-acting insulin analogues, which has now become much more familiar to clinicians than regular human insulin in general diabetes management.

Insulin analogs glulisine, aspart and lispro have been reported to have equal efficacy and in vivo potency compared to regular insulin in animals and humans, attributable to their similar receptor binding affinity and receptor mediated clearance (50-53), although only studies comparing the role of intravenous glulisine infusion as an alternative to intravenous infusion of regular human insulin has been performed (54). In spite of their more rapid onset, studies comparing the pharmacokinetics and pharmacodynamics of intravenous glulisine (an ultrashort-acting insulin analogue) to intravenous regular insulin (short-acting insulin) have found glulisine demonstrates equivalent glucose utilisation and disposal; and a similar distribution and elimination profile to regular insulin $(50,54)$.

Non-inferiority was demonstrated in a small, multicentre, open-label RCT $(n=68)$, comparing IV infusions of glulisine to regular human insulin. This study found both insulin types to be equally effective during the acute treatment of DKA, with no statistical difference in mean treatment duration $(15.7 \pm 4.5$ vs. $20.5 \pm 12 \mathrm{~h}$ ) or amount of insulin infused until resolution of DKA (70 \pm 33 vs. $76 \pm 46$ units) for glulisine and regular human insulin, respectively (54). Furthermore, the rate of decline of blood glucose concentrations, changes in bicarbonate and anion gap were similar, and neither groups experienced mortality or recurrence of DKA.

Due to the lack of superiority and the higher cost of insulin analogs, IV infusion of regular insulin is still considered the mainstay over insulin analogs in ADA consensus statement, though has not been specified in the NICE guidelines (1).

\section{Insulin-Route of Administration}

The attraction of subcutaneous insulin is to avoid the need for intensive care unit admission for IV insulin infusions and its associated high costs, although the concern is that it may not be as effective as IV insulin.

Studies comparing treatment of DKA using subcutaneous or intramuscular insulin against conventional IV insulin infusions found slower plasma glucose reductions at $1 \mathrm{~h}$ (failure to reduce by $10 \%)(55)$ and at $3 \mathrm{~h}$ (56) and slower ketone clearance (56).

Additionally, a retrospective study $(n=59)$ comparing bolus insulin injections in intravenous, subcutaneous, and intramuscular routes against continuous IV infusions found a higher rate of hypoglycemic episodes amongst the bolus insulin injection group ( 8 out of 30 patients), compared to the intravenous infusion group ( 1 out of 29 patients) ( $p=0.03)$ (57).

Importantly, none of the abovementioned studies assessed longer term outcomes such as time in intensive care or length of hospital stay. As such, it is unclear whether rapid correction of hyperglycemia and ketonaemia necessarily leads to better outcomes.

A Cochrane review in 2016 analysed five small RCTs (total $n=201$ ) comparing subcutaneous injections of insulin analogues (lispro and aspart) to regular insulin IV infusions in patients with mild to moderate DKA (Table 4) and revealed no statistical difference in time to resolution of DKA or rates of hypoglycaemia (42). These findings were based on very low to low-quality evidence and were limited by the number of included trials and participants. Other than hypoglycaemia, there was no analysis of adverse outcomes, and no deaths occurred in these studies (42).

The benefits of administering subcutaneous insulin injections hourly and 2 hourly outside of the intensive care setting 
must also be weighed against the increased demands on nursing staff on medical wards, increased variability with dose administration times, time to onset of action, peak effect, and duration of effect (58).

An alternative management strategy is the initiation of both an intravenous insulin and continuation of the patient's subcutaneous long-acting insulin during the initial management of DKA. This aims to decrease insulin infusion requirements, provide background insulin once intravenous insulin is discontinued, and reduce the risk of hypoglycaemia postcessation of insulin infusion. This was recommended in the NICE guidelines 2016 but was not present in the ADA 2009 consensus statement (1). This concept is supported by a prospective randomised study, which concluded that once daily subcutaneous insulin glargine administered during intravenous insulin infusions, safely prevented rebound hyperglycaemia without risk of hypoglycaemia (43).

Conversely, a pilot prospective RCT concluded that this model of treatment was feasible, although it did not find a significant benefit in time to closure of anion gap, or number of hypoglycaemic events (44) (Table 5).

Since the first trials in the 1970s, continuous subcutaneous insulin infusions delivered via insulin pumps have been used in patients with type 1 diabetes, and it is well established that DKA can occur as a complication of pump failure (59-62). Currently, there is no evidence surrounding the continuation or discontinuation of pump therapy during management of DKA. Current practice involves cessation of insulin pump therapy during management of DKA, then transition back to pump therapy after resolution of DKA (60). Conversely, given the potential roles of both continuous intravenous insulin and subcutaneous insulin injections, it could be postulated that insulin pumps may have a role in DKA management, as it is a convenient and pre-existing route of administration, although no studies have explored this potential.
The ADA 2009 consensus statement asserts that insulin therapy is effective regardless of route of administration, although both the ADA and NICE guidelines advocate for intravenous infusion $(1,29)$. The current evidence is weakly suggestive that there is no difference in outcome between administration of insulin as IV infusion or SC injections and is conflicting regarding impact of combining SC long-acting insulin with IV insulin infusion. Morbidity and safety data are limited with hypoglycemia being the only reported adverse outcome, and no deaths occurred in studies to date.

\section{Insulin-Role of Initial Intravenous Insulin Bolus}

An initial intravenous insulin bolus has been considered in multiple small trials.

A small prospective analysis in 1995 found patients who received an insulin bolus had a significantly higher incidence of hypoglycemia but no difference in hypokalaemia (57).

In contrast, a prospective observational cohort study in 2007 $(n=157)$ found no statistical difference in the incidence of hypoglycaemia, rate of glucose reduction, or length of hospital stay (63) and concluded that an initial bolus of intravenous insulin offered no significant clinical benefit or harm. The study was limited by lack of randomisation; standardised treatment protocols and used administration of 50\% dextrose as a surrogate definition for hypoglycemia rather than serum glucose levels.

In 2008, a small prospective randomised protocol established that an initial bolus of insulin avoided the need for supplemental insulin doses if the insulin infusion rate was less than 0.14 units/ $\mathrm{kg} / \mathrm{h}(1,64)$. The study compared patients $(n=12)$ who received a loading dose of 0.07 units $/ \mathrm{kg}$ of regular insulin, followed by $0.07 \mathrm{units} / \mathrm{kg} / \mathrm{h}$ insulin infusion, group $2(n=12)$ insulin infusion at $0.07 \mathrm{units} / \mathrm{kg} / \mathrm{h}$ without loading insulin and group $3(n=13)$ insulin infusion at $0.14 \mathrm{units} / \mathrm{kg} / \mathrm{h}$, without loading. It found that group 2 patients required supplemental insulin doses, whilst

TABLE 4 | Impact of insulin administration routes on diabetic ketoacidosis.

\begin{tabular}{|c|c|c|c|}
\hline Insulin types & Outcomes measured & Effect size & $\begin{array}{c}\text { Number of participants an } \\
\text { trials }\end{array}$ \\
\hline SC insulin lispro vs. IV regular insulin & Mean difference in time to resolution of DKA & $0.2 \mathrm{~h}(95 \% \mathrm{Cl}-1.7$ to 2.1$)(p=0.81)$ & ( $n=90,2$ trials $)$ \\
\hline SC insulin aspart vs. IV regular insulin & Mean difference in time to resolution of DKA & $-1 \mathrm{~h}(95 \% \mathrm{Cl}-3.2$ to 1.2$)(p=0.36)$ & $(n=30,1$ trial $)$ \\
\hline SC insulin lispro vs. IV regular insulin & Hypoglycemic events & Ratio of $0.59(95 \% \mathrm{Cl} 0.23-1.52)(p=0.28)$ & ( $n=156,4$ trials $)$ \\
\hline SC insulin aspart vs. IV regular insulin & Risk of hypoglycaemic episodes & Risk ratio 1.00 (95\% Cl 0.07-14.55) $(p=1.0)$ & $(n=30$, single trial) \\
\hline Insulin lispro vs. IV regular insulin & Difference in mean hospital length of stay & -0.4 days $(95 \% \mathrm{Cl}-1$ to 0.2$)(p=0.22)$ & ( $n=90,2$ trials) \\
\hline SC insulin aspart vs. IV regular insulin & Difference in mean length of stay & 1.1 days $(95 \% \mathrm{Cl}-3.3$ to 1.1$)(p=0.32)$ & $(n=30$, single trial) \\
\hline
\end{tabular}

Extracted from Cochrane Database of Systematic Reviews 2016 (42).

TABLE 5 | Outcomes of combining subcutaneous insulin with insulin infusion.

\begin{tabular}{|c|c|c|c|}
\hline Trial & Arms & $\begin{array}{l}\text { Outcomes of IV insulin infusion vs. IV insulin infusion } \\
\text { plus glargine retrospectively }\end{array}$ & $p$-Value \\
\hline $\begin{array}{l}\text { Hsia et al. - prospective randomised } \\
\text { study (43) }\end{array}$ & $\begin{array}{l}\text { IV Insulin infusion }(n=31) \text { vs. IV insulin } \\
\text { infusion plus glargine }{ }^{a}(n=30)\end{array}$ & $\begin{array}{l}\text { BGL > } 10 \text { mmol/L at } 12 \text { h follow-up: } 29 \text { (93.5\%) vs. } 10(33.3 \%) \\
\text { Hypoglycemic events: } 9.6 \text { vs. 0.0\% }\end{array}$ & $\begin{array}{l}<0.001 \\
\text { Not stated }\end{array}$ \\
\hline $\begin{array}{l}\text { Doshi et al. - pilot prospective } \\
\text { RCT (44) }\end{array}$ & $\begin{array}{l}\text { IV insulin infusion }(n=20) \text { vs. IV insulin } \\
\text { infusion plus glargine }(n=20)\end{array}$ & $\begin{array}{l}\text { Time to closure of anion gap (TCAG): } 11.6( \pm 6.4) \text { vs. } 10.2 \mathrm{~h}( \pm 6.8) \\
\text { Length of hospital stay: } 4.6 \text { ( } \pm 3.6) \text { vs. } 3.9 \text { days }( \pm 3.4) \\
\text { Number of hypoglycaemic events: } 3(15 \%) \text { vs. } 2(10 \%)\end{array}$ & $\begin{array}{l}0.63 \\
0.66 \\
1.0\end{array}$ \\
\hline
\end{tabular}

a Glargine dosed at $0.25 \mathrm{U} / \mathrm{kg}$ per body weight.

$R C T$, randomised controlled trial. 
other patients did not. Otherwise, there was no significant difference in duration taken to reach a serum resolution of DKA, length of hospital stay, hypokalaemia, or other complications including death (64).

Despite these findings, use of an initial bolus insulin dose has been incorporated into the ADA 2009 consensus statements but not in the NICE guidelines (29). The limited available evidence suggests use of bolus insulin results in no difference in outcomes.

\section{Insulin Dose}

Three decades ago, a small prospective RCT $(n=48)$ established the preference for low-dose insulin over high-dose insulin in management of hyperglycaemic emergencies (65). The study concluded there was no difference in correction rate of biochemical derangements, though $25-50 \%$ of patients who received high-dose insulin developed hypoglycaemia or hypokalaemia, compared to only $0-4 \%$ rate amongst patients who received lowdose insulin $(3,5,65)$.

The intravenous insulin rates remain contentious, with fixed weight-based insulin rates being advocated by national authorities $(1,5,24)$, to account for changing patient demographic, including obesity and higher insulin-resistance states such as pregnancy (5).

The Joint British Diabetes Society guidelines recommend insulin infusion at a rate that achieves a reduction in blood ketones by at least $0.5 \mathrm{mmol} / \mathrm{L} / \mathrm{h}$ or alternatively, a venous bicarbonate increase by $3 \mathrm{mmol} / \mathrm{L} / \mathrm{h}$ and capillary blood glucose reduction by $3 \mathrm{mmol} / \mathrm{L} / \mathrm{h}$ (5). Similarly, the 2009 ADA consensus statement recommend an insulin infusion rate adjusted to target a serum glucose reduction rate of 3-4 $\mathrm{mmol} / \mathrm{L} / \mathrm{h}$ with no suggestions for target ketone reduction (1), once serum glucose levels reach $11.1 \mathrm{mmol} / \mathrm{L}$, the insulin infusion rate should be reduced to $0.02-0.05$ units $/ \mathrm{kg} / \mathrm{h}$, coinciding with the addition of $5 \%$ dextrose infusion (1).

Whilst weak evidence has supported the traditional preference of low-dose over high-dose insulin, there is currently a lack of head to head studies guiding choice between fixed weight-based dosing over BGL-based sliding scale dosing.

\section{Transition to Subcutaneous Insulin}

On resolution of DKA and cessation of intravenous insulin infusion, rebound hyperglycaemia is a major issue $(43,66)$. Once an intravenous infusion is ceased its duration of action is only minutes, whilst the onset of basal subcutaneous insulin is $1-2 \mathrm{~h}$ and thus overlap of the two is an integral component of successful transition.

In 2007, a small study $(n=75)$ investigating the optimal insulin dose for transition from IV insulin infusion to subcutaneous insulin glargline, randomized patients to receive either $40 \%, 60 \%$, or $80 \%$ of their total daily insulin requirement, calculated from the rate of insulin infused during the last $6 \mathrm{~h}$ of their insulin infusion, as insulin glargine (67). The study concluded that insulin glargine administered at $80 \%$ of the total daily insulin requirement resulted in the highest percentage of capillary blood glucose level within target glycaemic range of $4.4-8.3 \mathrm{mmol} / \mathrm{L}$ within the initial $24 \mathrm{~h}$ after transition (67).
A prospective randomised trial compared multidose insulin regimens demonstrated that split mixed insulin $(\mathrm{NPH}$ plus regular insulin) and basal bolus insulin regimen (glargine and glulisine) offered similar glycaemic control though basal bolus regimen was associated with a lower rate of hypoglycaemic events (15 vs. $41 \%)$ (1).

The ADA 2009 consensus statement recommend patients normally on subcutaneous insulin should be recommenced on their usual dose if this was typically adequate for glycaemic control, whilst insulin naive patients should be commenced on either a multidose insulin of 2-3 boluses daily or basal bolus regimen (1).

Current limited evidence suggest that transitioning from insulin infusion to subcutaneous insulin using $80 \%$ of the total insulin dose offers optimal glycaemic control, with nil evidence to guide recommendations for either a spit mixed or basal bolus regime.

\section{Glucose Infusion}

Glucose infusion is recommended when blood glucose levels falls below $10-14 \mathrm{mmol} / \mathrm{L}(1,5,29,68)$ to avoid hypoglycaemia $(3,5)$ and to reduce the development of ketosis $(3,5,29)$. However, the choice of these thresholds is somewhat arbitrary.

Regarding the choice of fluid for maintenance of adequate glycaemic control, $5 \%$ dextrose was compared to $10 \%$ dextrose in a small RCT $(n=17)$. This study found that $10 \%$ dextrose resulted in significantly lower level of ketonaemia and a higher level of hyperglycaemia but did not result in any benefits in correction of acidosis or bicarbonate levels (36).

There is currently lack of evidence guiding threshold blood glucose levels for introduction of dextrose with no evidence suggesting a difference in outcomes between administration of dextrose 5 and $10 \%$.

\section{Interpretation of DKA Correction Rate}

Studies have traditionally used time to correction of DKA as a surrogate end point for patient outcomes, where resolution of DKA is defined as BGL $<11 \mathrm{mmol} / \mathrm{L}$ and two of the following criteria: serum bicarbonate level $\geq 15 \mathrm{mmol} / \mathrm{L}$, venous $\mathrm{pH}>7.3$ and a calculated anion gap $\leq 12 \mathrm{mmol} / \mathrm{L}$ (2). Despite the assumption that faster correction of DKA leads to better outcomes with shorter intensive care and hospital admission, there have been no studies which specifically establish this relationship. A prospective trial $(n=114)$ concluded that very low-dose insulin administration and slow re-equilibration were the fundamental strategies in optimal management (69). The study used a basal insulin infusion of $1 \mathrm{unit} / \mathrm{h}$, with a maximal decrease of blood glucose level of $3 \mathrm{mmol} / \mathrm{L} / \mathrm{h}$, reaching a mean blood glucose of $14 \mathrm{mmol} / \mathrm{L}$ at $12 \mathrm{~h}$, whilst rehydrating with $1 \mathrm{~L} / \mathrm{h}$ for the first $4 \mathrm{~h}$. This approach resulted in no mortality or lasting deficiencies in a small cohort (69).

Studies addressing this issue have been performed in the paediatric population and one retrospective observational study ( $n=67$ ) found an insulin infusion rate of 0.05 units $/ \mathrm{kg} / \mathrm{h}$ resulted in more gradual reduction in effective plasma osmolality over the first $12 \mathrm{~h}$, and a similar length of intensive care admission to patients who received insulin doses of 0.1 unit $/ \mathrm{kg} / \mathrm{h}(70)$. Similarly, a small open-label randomised trial $(n=50)$ also in the paediatric population found that standard insulin doses of 
$0.1 \mathrm{unit} / \mathrm{kg} / \mathrm{h}$ compared to low dose 0.05 units $/ \mathrm{kg} / \mathrm{h}$ was associated with higher rates of hypoglycaemia (20 vs. $4 \%, p=0.17$ ), hypokalaemia (48 vs. $20 \%, p=0.07$ ), and cerebral oedema (4 vs. 0\%), whilst time to reduction of blood glucose level and resolution of acidosis were similar (71).

Given these findings, we conclude that rapid resolution of DKA does not necessarily correlate with superior clinical outcomes. Guidelines do not address this issue, and further research is needed to explore this question.

\section{Use of Protocols}

Limited evidence suggests that the use of a protocol, whether based on national guidelines or individual institution-formed guidelines leads to a better adherence to key treatment principles and improved patient outcome $(4,12,46,72-77)$.

Adherence rates of DKA management protocols were studied, in a retrospective review of 71 cases. The study found an average of $30 \%$ adherence rates to guidelines and the study concluded outcomes were similar amongst patients treated with and without guidelines (78) (Table 6).

The uptake of a new DKA protocol was studied in a retrospective audit of 46 DKA episodes over a 4-month period in 2008, with adherence rates above 70\% in the initial hour (Table 6), although this declined after $1 \mathrm{~h}$ following commencement of treatment, resulting in incorrect fluids being administered and neglect in monitoring electrolytes (4). The reasons for this deviation from protocol were unclear although it was postulated to be due to lack of defined parameters indicating need for HDU referral, use of physician clinical judgement and inefficient patient handover during patient ward transfer and handover between shifts $(4,78)$.
Other authors raised the view that strict adherence to protocols may not be the gold-standard, and that deviations from protocols may be beneficial or even necessary in some instances (72). Protocol use may be poor if they are perceived to lack credibility, have systemic bias, reduce physician autonomy, or if they are not seen to be applicable to all patients $(4,46,72,73)$.

Regarding protocol impact on DKA treatment outcomes, a retrospective study compared patients managed with and without local protocol, found that patients treated with a local protocol had a significantly shorter mean time to normalisation of serum bicarbonate, lower incidences of hypokalaemia, and lower incidence of hypoglycaemia, and no significant difference in total insulin dose (11) (Table 7).

In 2011, a retrospective review of patients treated for DKA or HHS found that patients treated according to protocol had a shorter mean duration of time to resolution, and no difference in frequency of hypoglycaemia or hypokalaemia (12) (Table 7).

The limited current evidence suggest that adherence to protocols is poor to modest. Despite limited adherence to strict protocol fidelity, there appear to be improvements in patient outcomes $(4,12,46,72-74)$. Protocols are likely to be beneficial as a guide to nursing and medical staff with the caveat that deviation from protocol to tailor to individual circumstances may be warranted.

\section{LIMITATIONS}

We note this is a narrative review, not a systematic review, hence potential remains for further synthesis of identified evidence.

TABLE 6 | Adherence rates to diabetic ketoacidosis management protocols.

\begin{tabular}{|c|c|c|c|}
\hline Study & Cases & End point & Performance rates (\%) \\
\hline \multirow{3}{*}{$\begin{array}{l}\text { Singh et al. - retrospective } \\
\text { review (78) }\end{array}$} & \multirow[t]{3}{*}{$n=71$} & Timely initiation of intravenous fluid replacement & 30 \\
\hline & & Timely initiation of intravenous insulin & 31 \\
\hline & & Adequate intravenous fluids within the first $24 \mathrm{~h}$ & 30 \\
\hline \multirow{7}{*}{$\begin{array}{l}\text { Devalia-retrospective case } \\
\text { note review (4) }\end{array}$} & \multirow[t]{7}{*}{$n=46$} & DKA correctly diagnosed as per protocol & 78 \\
\hline & & Intravenous access and correct blood tests within the first hour of admission & 100 \\
\hline & & Appropriate fluid resuscitation & 87 \\
\hline & & Correct insulin prescribed & 72 \\
\hline & & Correct sliding scale & 73 \\
\hline & & Patients not requiring high dependency unit managed in the appropriate ward setting & 89 \\
\hline & & Patients requiring high dependency unit and appropriately referred & 46 \\
\hline
\end{tabular}

TABLE 7 | Impact on outcomes when treating diabetic ketoacidosis according to protocol.

\begin{tabular}{|c|c|c|c|c|}
\hline \multirow[t]{2}{*}{ Study } & \multirow[t]{2}{*}{ Arms } & \multirow[t]{2}{*}{ End points } & Findings & \multirow[t]{2}{*}{$p$-Value } \\
\hline & & & $\begin{array}{l}\text { Protocol vs. non-protocol arms } \\
\text { respectively }\end{array}$ & \\
\hline \multirow{4}{*}{$\begin{array}{l}\text { Thuzar et al. - retrospective } \\
\text { study (11) }\end{array}$} & Protocol $(n=35)$ & Mean length of hospitalisation & 37.9 vs. $49.2 \mathrm{~h}$ & 0.01 \\
\hline & No protocol $(n=36)$ & Mean time to normalisation of serum bicarbonate & 15.1 vs. $24.6 \mathrm{~h}$ & 0.01 \\
\hline & & Incidences of hypokalaemia & 28.6 vs. $52.8 \%$ & 0.038 \\
\hline & & Incidence of hypoglycaemia & 8.6 vs. $28 \%$ & 0.036 \\
\hline \multirow{4}{*}{$\begin{array}{l}\text { Hara et al. - retrospective } \\
\text { review (12) }\end{array}$} & Protocol $(n=113)$ & Mean difference in time to resolution & -9.2 h (95\% Cl: 4.7-13.7) & $<0.01$ \\
\hline & Non-protocol $(n=143)$ & Frequency of hypoglycaemia & 8 vs. $11.2 \%$ & 0.259 \\
\hline & & Frequency of hypokalaemia & 30.1 vs. $32.3 \%$ & 0.413 \\
\hline & & Mean difference in length of hospital stay & -2.04 days (95\% Cl: 0.61-3.46) & 0.005 \\
\hline
\end{tabular}


Although we considered several key aspects of DKA management, there are many other aspects of treatment that were not formally reviewed. These aspects include the roles and implications of separate emergency department, ward, and intensive care unit protocols; severity assessment; special patient groups with comorbidities including drug and alcohol use; age-based fluid replacement, patient education and other preventative strategies, and complications of DKA. Euglycaemic DKA was also not reviewed.

\section{DISCUSSION AND CONCLUSION}

On review, the strength and breadth of original evidence is limited. Current weak evidence suggests crystalloids are preferred over colloids. There is no clear evidence for superiority of a given crystalloid nor for a given rate of rehydration.

Low-dose insulin has limited evidence for superior safety over high-dose insulin. Administering bolus insulin prior to low-dose insulin infusions $<0.14$ units $/ \mathrm{kg} / \mathrm{h}$ may obviate the need for additional insulin supplementation. Intravenous regular insulin infusions are traditionally preferred, with emerging evidence for non-inferiority of subcutaneous insulin and with no head to head comparisons between fixed weight-based versus blood glucose-based insulin dosing regimens. Clear evidence is lacking for timing of initiation and titration of dextrose infusions, in addition to replacement of potassium, phosphate, bicarbonate. Traditionally, studies have used mean time to resolution of DKA as favourable end points, although this correlation has no supporting evidence.

\section{REFERENCES}

1. Kitabchi AE, Umpierrez GE, Miles JM, Fisher JN. Hyperglycemic crises in adult patients with diabetes. Diabetes Care (2009) 32(7):1335-43. doi:10.2337/ dc09-1563

2. Nyenwe EA, Kitabchi AE. The evolution of diabetic ketoacidosis: an update of its etiology, pathogenesis and management. Metabolism (2016) 65(4):507-21. doi:10.1016/j.metabol.2015.12.007

3. Nyenwe EA, Kitabchi AE. Evidence-based management of hyperglycemic emergencies in diabetes mellitus. Diabetes Res Clin Pract (2011) 94(3):340-51. doi:10.1016/j.diabres.2011.09.012

4. Devalia B. Adherance to protocol during the acute management of diabetic ketoacidosis: would specialist involvement lead to better outcomes? Int J Clin Pract (2010) 64(11):1580-2. doi:10.1111/j.1742-1241.2010.02348.x

5. Savage M, Dhatariya K, Kilvert A, Rayman G, Rees J, Courtney C, et al. Joint British Diabetes Societies guideline for the management of diabetic ketoacidosis. Diabet Med (2011) 28(5):508-15. doi:10.1111/j.1464-5491.2011. 03246.x

6. Welfare AIoHa. In: Government Australia, editor. Diabetes among Young Australians. Canberra, ACT: AIHW (2012).

7. Dhatariya K. The management of Diabetic Ketoacidosis in Adults. UK: Joint British Diabetes Society (2013).

8. Azevedo LC, Choi H, Simmonds K, Davidow J, Bagshaw SM. Incidence and long-term outcomes of critically ill adult patients with moderate-to-severe diabetic ketoacidosis: retrospective matched cohort study. J Crit Care (2014) 29(6):971-7. doi:10.1016/j.jcrc.2014.07.034

9. Carr ME. Diabetes mellitus: a hypercoagulable state. J Diabetes Complications (2001) 15(1):44-54. doi:10.1016/S1056-8727(00)00132-X

10. Hamblin P, Topliss D, Chosich N, Lording D, Stockigt J. Deaths associated with diabetic ketoacidosis and hyperosmolar coma. 1973-1988. Med J Aust (1989) 151(8): 439, 41-2, 44.
Few studies consider patient outcome using various protocols, with improvement in outcomes.

Currently, there is a major deficiency of strong evidence for the optimal management of DKA in the adult population with a mixture of consensus opinion and weak evidence providing the framework within which physicians manage DKA. There are inherent difficulties in conducting robust studies and these include low rates of patient admissions with DKA, large numbers of patients required to show differences and the multiple confounding variables contributing to outcomes. Despite this, all components of DKA management would benefit from prospective RCTs and there is vast potential for further studies to elucidate or establish a more robust evidence-based practice for best patient outcomes.

\section{AUTHOR CONTRIBUTIONS}

TT and AP-selection of articles and drafting of manuscript. TT, AP, EE, AW, JZ, JM, and RB-all authors contributed substantially to the conception and design of the work, reviewing, final approvals of the version submitted, and agree to be accountable for accuracy and integrity of content.

\section{FUNDING}

EE was supported by an NHMRC Early Career Fellowship, a Viertel Clinical Investigatorship, RACP Fellowship and Sir Edward Weary Dunlop Medical Research Foundation research grant.

11. Thuzar M, Malabu UH, Tisdell B, Sangla KS. Use of a standardised diabetic ketoacidosis management protocol improved clinical outcomes. Diabetes Res Clin Pract (2014) 104(1):e8-11. doi:10.1016/j.diabres.2014.01.016

12. Hara JS, Rahbar AJ, Jeffres MN, Izuora KE. Impact of a hyperglycemic crises protocol. Endocr Pract (2013) 19(6):953-62. doi:10.4158/EP13077.OR

13. Gosmanov AR, Gosmanova EO, Dillard-Cannon E. Management of adult diabetic ketoacidosis. Diabetes Metab Syndr Obes (2014) 7:255-64. doi:10.2147/ DMSO.S50516

14. Chua HR, Venkatesh B, Stachowski E, Schneider AG, Perkins K, Ladanyi S, et al. Plasma-Lyte 148 vs $0.9 \%$ saline for fluid resuscitation in diabetic ketoacidosis. J Crit Care (2012) 27(2):138-45. doi:10.1016/j.jcrc.2012. 01.007

15. Perel P, Roberts I, Ker K. Colloids versus crystalloids for fluid resuscitation in critically ill patients. Cochrane Database Syst Rev (2013) 2:CD000567. doi:10.1002/14651858.CD000567.pub6

16. Mahler SA, Conrad SA, Wang H, Arnold TC. Resuscitation with balanced electrolyte solution prevents hyperchloremic metabolic acidosis in patients with diabetic ketoacidosis. Am J Emerg Med (2011) 29(6):670-4. doi:10.1016/j. ajem.2010.02.004

17. Orbegozo Cortes D, Gamarano Barros T, Njimi H, Vincent JL. Crystalloids versus colloids: exploring differences in fluid requirements by systematic review and meta-regression. Anesth Analg (2015) 120(2):389-402. doi:10.1213/ ANE.0000000000000564

18. Guidet B, Soni N, Della Rocca G, Kozek S, Vallet B, Annane D, et al. A balanced view of balanced solutions. Crit Care (2010) 14(5):1. doi:10.1186/ cc9230

19. Zarychanski R, Abou-Setta AM, Turgeon AF, Houston BL, McIntyre L, Marshall JC, et al. Association of hydroxyethyl starch administration with mortality and acute kidney injury in critically ill patients requiring volume resuscitation: a systematic review and meta-analysis. JAMA (2013) 309(7):678-88. doi:10.1001/jama.2013.430 
20. Oh MS, Banerji MA, Carroll HJ. The mechanism of hyperchloremic acidosis during the recovery phase of diabetic ketoacidosis. Diabetes (1981) 30(4):310-3. doi:10.2337/diabetes.30.4.310

21. Oh MS, Carroll HJ, Goldstein DA, Fein IA. Hyperchloremic acidosis during the recovery phase of diabetic ketosis. Ann Intern Med (1978) 89(6):925-7. doi:10.7326/0003-4819-89-6-925

22. Adrogue HJ, Barrero J, Eknoyan G. Salutary effects of modest fluid replacement in the treatment of adults with diabetic ketoacidosis. Use in patients without extreme volume deficit. JAMA (1989) 262(15):2108-13. doi:10.1001/ jama.1989.03430150076029

23. Waldhausl W, Kleinberger G, Korn A, Dudczak R, Bratusch-Marrain P, Nowotny P. Severe hyperglycemia: effects of rehydration on endocrine derangements and blood glucose concentration. Diabetes (1979) 28(6):577-84. doi:10.2337/diab.28.6.577

24. Kitabchi AE, Umpierrez GE, Murphy MB, Barrett EJ, Kreisberg RA, Malone JI, et al. Management of hyperglycemic crises in patients with diabetes. Diabetes Care (2001) 24(1):131-53. doi:10.2337/diacare.24.1.131

25. Kitabchi AE, Umpierrez GE, Fisher JN, Murphy MB, Stentz FB. Thirty years of personal experience in hyperglycemic crises: diabetic ketoacidosis and hyperglycemic hyperosmolar state. J Clin Endocrinol Metab (2008) 93(5):1541-52. doi:10.1210/jc.2007-2577

26. Van Zyl DG, Rheeder P, Delport E. Fluid management in diabetic-acidosis Ringer's lactate versus normal saline: a randomized controlled trial. QJM (2011) 105(4):337-43. doi:10.1093/qjmed/hcr226

27. Yunos NM, Bellomo R, Hegarty C, Story D, Ho L, Bailey M. Association between a chloride-liberal vs chloride-restrictive intravenous fluid administration strategy and kidney injury in critically ill adults. JAMA (2012) 308(15):1566-72. doi:10.1001/jama.2012.13356

28. Owen OE, Licht JH, Sapir DG. Renal function and effects of partial rehydration during diabetic ketoacidosis. Diabetes (1981) 30(6):510-8. doi:10.2337/ diab.30.6.510

29. NICE. Type 1 Diabetes: Diagnosis and Management. UK: NICE guideline (2015).

30. Durward A, Ferguson LP, Taylor D, Murdoch IA, Tibby SM. The temporal relationship between glucose-corrected serum sodium and neurological status in severe diabetic ketoacidosis. Arch Dis Child (2011) 96(1):50-7. doi:10.1136/ adc.2009.170530

31. Hoffman WH, Steinhart CM, el Gammal T, Steele S, Cuadrado AR, Morse PK. Cranial CT in children and adolescents with diabetic ketoacidosis. AJNR Am J Neuroradiol (1988) 9(4):733-9.

32. Sterns RH. Disorders of plasma sodium - causes, consequences, and correction. N Engl J Med (2015) 372(1):55-65. doi:10.1056/NEJMra1404489

33. Edge JA, Jakes RW, Roy Y, Hawkins M, Winter D, Ford-Adams ME, et al. The UK case-control study of cerebral oedema complicating diabetic ketoacidosis in children. Diabetologia (2006) 49(9):2002-9. doi:10.1007/s00125-0060363-8

34. Azzopardi J, Gatt A, Zammit A, Alberti G. Lack of evidence of cerebral oedema in adults treated for diabetic ketoacidosis with fluids of different tonicity. Diabetes Res Clin Pract (2002) 57(2):87-92. doi:10.1016/ S0168-8227(02)00019-0

35. Laggner AN, Lenz K, Kleinberger G, Sommer G, Druml W, Schneeweiss B. Influence of fluid replacement on extravascular lung water (EVLW) in patients with diabetic ketoacidosis. Intensive Care Med (1988) 14(3):201-5. doi:10.1007/BF00717987

36. Krentz A, Hale P, Singh B, Nattrass M. The effect of glucose and insulin infusion on the fall of ketone bodies during treatment of diabetic ketoacidosis. Diabet Med (1989) 6(1):31-6. doi:10.1111/j.1464-5491.1989.tb01135.x

37. Alonso K. Immediate plasma potassium levels in treating diabetic ketoacidosis. Arch Intern Med (1988) 148(3):750-2. doi:10.1001/archinte.148. 3.750

38. Barsotti MM. Potassium phosphate and potassium chloride in the treatment of DKA. Diabetes Care (1980) 3(4):569. doi:10.2337/diacare.3.4.569a

39. Fulop M. Serum potassium in lactic acidosis and ketoacidosis. N Engl J Med (1979) 300(19):1087-9. doi:10.1056/NEJM197905103001905

40. Walsh C, Soler N, James H, Fitzgerald M, Malins J. Studies on whole-body potassium in non-ketoacidotic diabetics before and after treatment. $\mathrm{Br} \mathrm{Med} \mathrm{J}$ (1974) 4(5947):738-40. doi:10.1136/bmj.4.5947.738
41. Palmer BF, Clegg DJ. Electrolyte and acid-base disturbances in patients with diabetes mellitus. N Engl J Med (2015) 373(6):548-59. doi:10.1056/ NEJMra1503102

42. Andrade-Castellanos CA, Colunga-Lozano LE, Delgado-Figueroa N, Gonzalez-Padilla DA. Subcutaneous rapid-acting insulin analogues for diabetic ketoacidosis. Cochrane Libr (2016) (1):CD011281. doi:10.1002/14651858. CD011281.pub2

43. Hsia E, Seggelke S, Gibbs J, Hawkins RM, Cohlmia E, Rasouli N, et al. Subcutaneous administration of glargine to diabetic patients receiving insulin infusion prevents rebound hyperglycemia. JClin Endocrinol Metab (2012) 97(9):3132-7. doi:10.1210/jc.2012-1244

44. Doshi P, Potter AJ, De Los Santos D, Banuelos R, Darger BF, Chathampally Y. Prospective randomized trial of insulin glargine in acute management of diabetic ketoacidosis in the emergency department: a pilot study. Acad Emerg Med (2015) 22(6):657-62. doi:10.1111/acem.12673

45. Morris LR, MURPHY MB, KITABCHI AE. Bicarbonate therapy in severe diabetic ketoacidosis. Ann Intern Med (1986) 105(6):836-40. doi:10.7326/0003-4819-105-6-836

46. Jervis A, Champion S, Figg G, Langley J, Adams GG. Prevalence of diabetes ketoacidosis rises and still no strict treatment adherence. Curr Diabetes Rev (2013) 9(1):54-61. doi:10.2174/157339913804143199

47. Burghen GA, Etteldorf JN, Fisher JN, Kitabchi AQ. Comparison of high-dose and low-dose insulin by continuous intravenous infusion in the treatment of diabetic ketoacidosis in children. Diabetes Care (1980) 3(1):15-20. doi:10.2337/diacare.3.1.15

48. Shankar V, Haque A, Churchwell KB, Russell W. Insulin glargine supplementation during early management phase of diabetic ketoacidosis in children. Intensive Care Med (2007) 33(7):1173-8. doi:10.1007/s00134-007-0674-3

49. Storms FE, Lutterman JA, van't Laar A. Comparison of efficacy of human and porcine insulin in treatment of diabetic ketoacidosis. Diabetes Care (1987) 10(1):49-55. doi:10.2337/diacare.10.1.49

50. Barski L, Kezerle L, Zeller L, Zektser M, Jotkowitz A. New approaches to the use of insulin in patients with diabetic ketoacidosis. Eur J Intern Med (2013) 24(3):213-6. doi:10.1016/j.ejim.2013.01.014

51. Gillies PS, Figgitt DP, Lamb HM. Insulin glargine. Drugs (2000) 59(2):253-60. doi:10.2165/00003495-200059020-00009

52. Hennige A, Strack V, Metzinger E, Seipke G, Häring H-U, Kellerer M. Effects of new insulin analogues HMR1964 (insulin glulisine) and HMR1423 on insulin receptors. Diabetologia (2005) 48(9):1891-7. doi:10.1007/s00125005-1870-8

53. Plum A, Agersø $\mathrm{H}$, Andersen L. Pharmacokinetics of the rapid-acting insulin analog, insulin aspart, in rats, dogs, and pigs, and pharmacodynamics of insulin aspart in pigs. Drug Metab Dispos (2000) 28(2):155-60.

54. Umpierrez GE, Jones S, Smiley D, Mulligan P, Keyler T, Temponi A, et al. Insulin analogs versus human insulin in the treatment of patients with diabetic ketoacidosis A randomized controlled trial. Diabetes Care (2009) 32(7):1164-9. doi:10.2337/dc09-0169

55. Umpierrez GE, Latif K, Stoever J, Cuervo R, Park L, Freire AX, et al. Efficacy of subcutaneous insulin lispro versus continuous intravenous regular insulin for the treatment of patients with diabetic ketoacidosis. Am JMed (2004) 117(5):291-6. doi:10.1016/j.amjmed.2004.05.010

56. Ersöz H, Ukinc K, Köse M, Erem C, Gunduz A, Hacihasanoglu A, et al. Subcutaneous lispro and intravenous regular insulin treatments are equally effective and safe for the treatment of mild and moderate diabetic ketoacidosis in adult patients. Int JClin Pract (2006) 60(4):429-33. doi:10.1111/j.1368-5031.2006.00786.x

57. Butkiewicz EK, Leibson CL, O’Brien PC, Palumbo PJ, Rizza RA. Insulin therapy for diabetic ketoacidosis: bolus insulin injection versus continuous insulin infusion. Diabetes Care (1995) 18(8):1187-90. doi:10.2337/diacare. 18.8.1187

58. Umpierrez GE, Cuervo R, Karabell A, Latif K, Freire AX, Kitabchi AE. Treatment of diabetic ketoacidosis with subcutaneous insulin aspart. Diabetes Care (2004) 27(8):1873-8. doi:10.2337/diacare.27.8.1873

59. White D, Waugh N, Elliott J, Lawton J, Barnard K, Campbell MJ, et al. The Relative Effectiveness of Pumps Over MDI and Structured Education (REPOSE): study protocol for a cluster randomised controlled trial. BMJ Open (2014) 4(9):e006204. doi:10.1136/bmjopen-2014-006204 
60. Houlden RL, Moore S. In-hospital management of adults using insulin pump therapy. Can J Diabetes (2014) 38(2):126-33. doi:10.1016/j.jcjd.2014. 01.011

61. Goguen J, Gilbert J. Canadian Diabetes Association 2013 Clinical Practice Guidelines for the Prevention and Management of Diabetes in Canada: Hyperglycemic Emergencies in Adults. Canadian Diabetes Association (2013).

62. Ludvigsson J, Samuelsson U. Continuous insulin infusion (CSII) or modern type of multiple daily injections (MDI) in diabetic children and adolescents a critical review on a controversial issue. Pediatr Endocrinol Rev (2007) 5(2):666-78.

63. Goyal N, Miller JB, Sankey SS, Mossallam U. Utility of initial bolus insulin in the treatment of diabetic ketoacidosis. J Emerg Med (2010) 38(4):422-7. doi:10.1016/j.jemermed.2007.11.033

64. Kitabchi AE, Murphy MB, Spencer J, Matteri R, Karas J. Is a priming dose of insulin necessary in a low-dose insulin protocol for the treatment of diabetic ketoacidosis? Diabetes Care (2008) 31(11):2081-5. doi:10.2337/ dc08-0509

65. Kitabchi AE, Ayyagari V, Guerra SM. The efficacy of low-dose versus conventional therapy of insulin for treatment of diabetic ketoacidosis. Ann Intern Med (1976) 84(6):633-8. doi:10.7326/0003-4819-84-6-633

66. Czosnowski QA, Swanson JM, Lobo BL, Broyles JE, Deaton PR, Finch CK. Evaluation of glycemic control following discontinuation of an intensive insulin protocol. J Hosp Med (2009) 4(1):28-34. doi:10.1002/ jhm.393

67. Schmeltz L, DeSantis A, Schmidt K, O'Shea-Mahler E, Rhee C, Brandt S, et al. Conversion of intravenous insulin infusions to subcutaneously administered insulin glargine in patients with hyperglycemia. Endocr Pract (2007) 12(6):641-50. doi:10.4158/EP.12.6.641

68. Goguen J, Gilbert J. Hyperglycemic emergencies in adults. Can JDiabetes (2013) 37(Suppl 1):S72-6. doi:10.1016/j.jcjd.2013.01.023

69. Wagner A, Risse A, Brill H-L, Wienhausen-Wilke V, Rottmann M, Sondern K, et al. Therapy of severe diabetic ketoacidosis. Zero-mortality under very-low-dose insulin application. Diabetes Care (1999) 22(5):674-7. doi:10.2337/diacare.22.5.674

70. Al Hanshi S, Shann F. Insulin infused at 0.05 versus 0.1 units $/ \mathrm{kg} / \mathrm{hr}$ in children admitted to intensive care with diabetic ketoacidosis. Pediatr Crit Care Med (2011) 12(2):137-40. doi:10.1097/PCC.0b013e3181e2a21b

71. Nallasamy K, Jayashree M, Singhi S, Bansal A. Low-dose vs standard-dose insulin in pediatric diabetic ketoacidosis: a randomized clinical trial. JAMA Pediatr (2014) 168(11):999-1005. doi:10.1001/jamapediatrics. 2014.1211

72. Oeyen S. About protocols and guidelines: it's time to work in harmony! Crit Care Med (2007) 35(1):292-3. doi:10.1097/01.CCM.0000251495.83633.D7

73. Bull SV, Douglas IS, Foster M, Albert RK. Mandatory protocol for treating adult patients with diabetic ketoacidosis decreases intensive care unit and hospital lengths of stay: results of a nonrandomized trial. Crit Care Med (2007) 35(1):41-6. doi:10.1097/01.CCM.0000249825.18677.D2

74. Ilag LL, Kronick S, Ernst RD, Grondin L, Alaniz C, Liu L, et al. Impact of a critical pathway on inpatient management of diabetic ketoacidosis. Diabetes Res Clin Pract (2003) 62(1):23-32. doi:10.1016/S0168-8227(03)00143-8

75. Braithwaite DT, Umpierrez GE, Braithwaite SS. A quadruply-asymmetric sigmoid to describe the insulin-glucose relationship during intravenous insulin infusion. J Healthc Eng (2014) 5(1):23-53. doi:10.1260/2040-2295. 5.1 .23

76. Fort A, Narsinghani U, Bowyer F. Evaluating the safety and efficacy of Glucommander, a computer-based insulin infusion method, in management of diabetic ketoacidosis in children, and comparing its clinical performance with manually titrated insulin infusion. J Pediatr Endocrinol Metab (2009) 22(2):119-25. doi:10.1515/JPEM.2009.22.2.119

77. Devi R, Zohra T, Howard BS, Braithwaite SS. Target attainment through algorithm design during intravenous insulin infusion. Diabetes Technol Ther (2014) 16(4):208-18. doi:10.1089/dia.2013.0287

78. Singh RK, Perros P, Frier BM. Hospital management of diabetic ketoacidosis: are clinical guidelines implemented effectively? Diabet Med (1997) 14(6):482-6. doi:10.1002/(SICI)1096-9136(199706)14:6<482::AID-DIA371> 3.3.CO;2-1

Conflict of Interest Statement: The authors declare that the research was conducted in the absence of any commercial or financial relationships that could be construed as a potential conflict of interest.

Copyright (c) 2017 Tran, Pease, Wood, Zajac, Mårtensson, Bellomo and Ekinci. This is an open-access article distributed under the terms of the Creative Commons Attribution License (CC BY). The use, distribution or reproduction in other forums is permitted, provided the original author(s) or licensor are credited and that the original publication in this journal is cited, in accordance with accepted academic practice. No use, distribution or reproduction is permitted which does not comply with these terms. 